\title{
ORGANIC METALS FROM CHIRAL BEDT-TTF DONORS
}

\author{
Ben-ming Chen, * F. Deilacher, M. Hoch, H. J. Keller, and Pei-ji Wu* \\ Anorganisch-Chemisches Institut \\ Universität Heidelberg, Im Neuenheimer Feld 270 \\ D-6900 Heidelberg, GERMAN FEDERAL REPUBLIC \\ *Guests from Academia Sinica, Institute of Chemistry, Beijing, CHINA
}

\author{
S. Gärtner, S. Kahlich, and D. Schweitzer** \\ Max-Planck-Institut für Med. Forschung \\ AG.: Molekülkristalle, Jahnstr. 29 \\ D-6900 Heidelberg, GERMAN FEDERAL REPUBLIC \\ **3. Physikalisches Institut der Universität Stuttgart, Pfaffenwaldring 75 \\ D-7000 Stuttgart 80, GERMAN FEDERAL REPUBLIC
}

\section{STATUS QUO ANTE}

The chemistry and physics of solids with layered structures has been of central interest in the solid state sciences for several decades [1]. One of the many scientifically and technically important aspects connected with these materials is the possibility of pronounced two-dimensional delocalized interatomic and/or intermolecular electronic interactions, which lead to unusual magnetic and electric properties in the bulk. Especially remarkable are a variety of phase transitions, which occur in selected materials of this type at different temperatures. Typical, and very well-known, examples of such specimens are graphite, the binary sulfides $\mathrm{MoS}_{2}$ and $\mathrm{TaS}_{2}$, together with several of their intercalation compounds, ternary or quarternary chalcogenides and/or halides of the transition elements, and so on. Inorganic ternary systems, composed of anionic transition metal oxide layers and different kinds of countercations located between these anion sheets, are especially well-suited for systematic variations in composition and - as a consequence thereof - in solid state properties. Varying stages of band fillings can be achieved "chemically," depending on the number of electrons per metal ion in the different sheets. The systematic work on these materials culminated in the discovery of superconductivity above $40 \mathrm{~K}$ by Müller and Bednorz in layered copper oxide structures [2].

With the exception of graphite-related compounds - which consist mainly of carbon- there were no comparable classes of electronically collective interacting layered organic materials. Most of the two-dimensional organic solids - which means layered matrices made up mainly of the main group elements carbon, nitrogen, oxygen, hydrogen and sulfur - are diamagnetic and insulating. The electrically conducting and/or magnetically interesting organic charge transfer solids, on the other hand, contain columns or chains of interacting molecules or atoms. This arrangement results in pronounced "one-dimensional" physical properties, with the well-known phase transitions at comparatively high temperatures.

\section{STATUS QUO}

Up to now, there is one organic two-dimensional "exception:" the cation radical salts of the donor BEDT-TTF. Numerous BEDT-TTF salts - metallic, semiconducting and/or insulating - have been prepared in the last few years $[3,4]$, after Saito et al. firstly pointed to 
the typical two-dimensional molecular interactions in a radical salt of this donor [5]. Almost all of the solids, obtained so far by electrochemical oxidation of BEDT-TTF, in the presence of suitable counter anions, crystallize in two-dimensionally interacting arrays. The typical arrangement consists of sheets of anions - normally diamagnetic and not interacting with each other, nor with the BEDT-TTF partners - and layers of BEDT-TTF cations, in which the molecules can be oriented in quite different ways. There are often columns, or pairs of cations, with strong intermolecular S-S-interactions. Independent of these differences in molecular arrangement, the main feature of all these materials is a layered structure. The different orientations of the cation radicals in a layer with respect to each other, and in relation to the counter anion lattice, leads, in most cases, to strong two-dimensional, in other cases, to typical one-dimensional electronic interactions. Nevertheless, the general structure in most of the materials is of the layered type. This variety of [BEDT-TTF $]_{y^{n+}} \mathrm{X}^{\mathrm{n}-}$ structures shows that this material establishes a new class of two-dimensional organic matrices.

\section{THE PLAN}

The versatility of this organic counterpart of the well-known inorganic layered solids opens the possibility for systematic "chemical" variation of band fillings and electronic interactions. The redox properties of the different lattice elements can be varied systematically by chemical methods, since the BEDT-TTF salts are open to synthetic variations in both types of layers very similar to the famous inorganic ternary oxides. So far, most of the recent work to vary the physical properties by chemical action concentrated on the preparation of BEDT-TTF salts with a wide variety of anions. Fewer efforts were concerned with a systematic variation of the BEDT-TTF moieties [6]. One major problem arises in the pursuit of the latter goal: the substitution of the peripheral hydrogen atoms could lead to a manifold of chiral molecules.

\section{THE PROJECT}

For a beginning, we decided to substitute the hydrogen atoms of the peripheral ethylene groups of BEDT-TTF systematically by methyl groups. The preparation of these substituted BEDT-TTF donors is by no means trivial. The hydrogen methyl group exchange leads to different configurational isomers and, especially, to a variety of chiral molecules.

First, we concentrated our efforts on two symmetrical molecules, DIET and TMET.<smiles>CC1CSC2SC(=C3SC4=C(S3)S[C@@H](C)CS4)SC2S1</smiles>

DIET<smiles>C[C@H]1S[C@H]2SC(=C3S[C@H]4S[C@H](C)[C@@H](C)S[C@@H]4S3)S[C@@H]2S[C@@H]1C</smiles>

TMET

DIET can be arranged in a "trans" - or a "cis" - form.

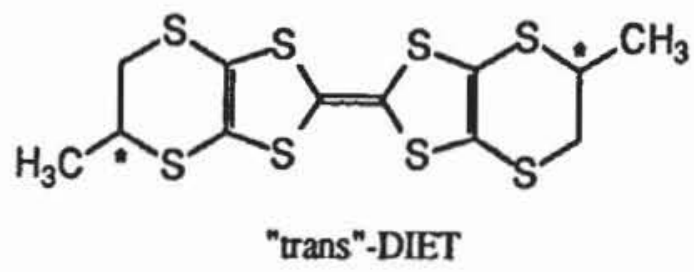

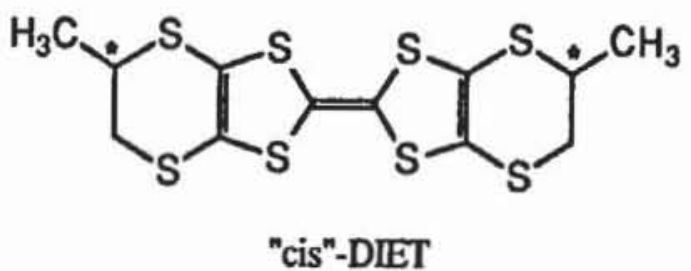

Each of these DIET isomers could occur in two enantiomers and an additional diastereomer. In the case of the tetramethyl derivative TMET, two pairs of enantiomers and three diastereomers could result from the preparation. 


\section{Chemistry}

The synthetic route to the different donors is in imitation of the usual procedure to obtain BEDT-TTF. A complex of the dithiolate I<smiles>CC(C)(C)N</smiles>

I

is reacted with 1,2-dibromopropane and 2,3-dibromobutane, respectively, to yield the thiones II and III<smiles>C[C@H]1CSc2sc(=S)sc2S1</smiles>

II<smiles>C[C@@H]1Sc2sc(=S)sc2S[C@@H]1C</smiles>

III

which are converted to the ketones IV and V<smiles>C[C@H]1CSc2sc(=O)sc2S1</smiles>

IV<smiles>C[C@H]1Sc2sc(=O)sc2S[C@@H]1C</smiles>

V

in order to be self-coupled by action of $\mathrm{P}(\mathrm{OR})_{3}$. A mixture of DIET and TMET isomers results. DIET has been obtained earlier by a similar procedure [6], but no details concerning its structure have been reported so far. TMET was isolated starting from a sulphate diester earlier, and some of its radical salts have been described [7].

In order to investigate the basic chemical and structural properties of the donors, we decided to electrocrystallize mixtures of the isomers of the donor, obtained by starting with racemic precursors. DIET salts crystallize with more ease at the electrode, compared to the TMET materials. The radical salts of the latter are very soluble in polar solvents, making the crystallization more difficult. Polar solvents - on the other hand - are necessary to dissolve the supporting electrolyte. In the case of DIET this difficulty is less pronounced. So far, we obtained well characterized "racemic" DIET solids with $\mathrm{FSO}_{3}{ }^{-}, \mathrm{ClO}_{4}^{-}, \mathrm{HSO}_{4}^{-}, \mathrm{NO}_{3}^{-}, \mathrm{I}_{3}{ }^{-}$, $\left[\mathrm{AuI}_{2}\right]^{-}, \mathrm{ReO}_{4}^{-}, \mathrm{PF}_{6}^{-}, \mathrm{IO}_{4}^{-}, \mathrm{SO}_{3} \mathrm{CF}_{3}^{-}$and $\mathrm{BF}_{4}^{-}$as counter anions.

\section{$\mathrm{X}$-ray Characterization}

Structural work on these materials is complicated by the fact that the crystals contain mixtures of different isomers, and are therefore disordered. Nevertheless, it could be shown that the donor DIET is obtained solely as the trans-isomer. Surprising is the comparatively long "central" $\mathrm{C}=\mathrm{C}$ bond $(1.4 \AA)$ of the DIET donor.

A typical example for the structural problems in the radical salts is given in Fig. 1. It shows the molecular structure of $(\mathrm{DIET})_{2}\left(\mathrm{FSO}_{3}\right)_{3}$. All the indicated atomic positions are statistically occupied. The same is valid for the isostructural $(\mathrm{DIET})_{2}\left(\mathrm{BF}_{4}\right)_{3}$. Nevertheless, the structure elucidation clearly reveals the $2: 3$ composition of both compounds, which results in an average +1.5 charge on each DIET cation. It is interesting to note that the central $\mathrm{C}=\mathrm{C}$ bond distances of $1.322(35) \AA$ in the former, and of $1.292(26) \AA$ in the latter material, are appreciably shortened with respect to these $\mathrm{C}=\mathrm{C}$ distances in the neutral donor. Relating to recently proposed models $[3,8]$, this trend would not be indicative of positive charges at all, 


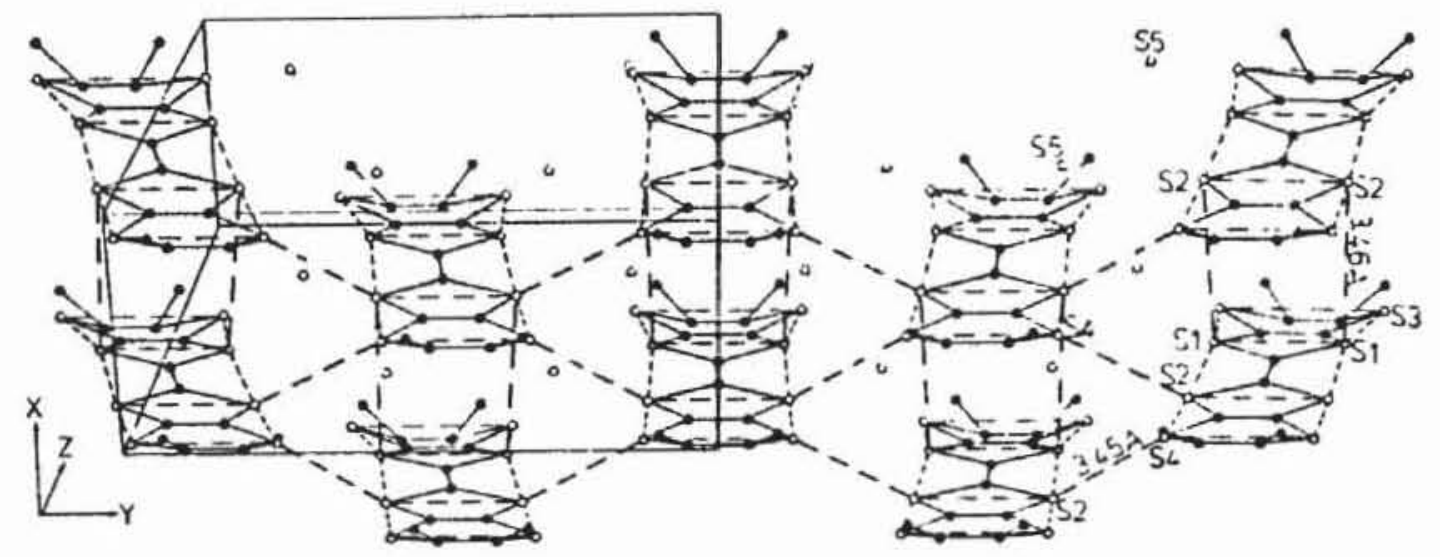

Fig. 1. Crystal structure of $(\text { DIET) })_{2}\left(\mathrm{FSO}_{3}\right)_{3}$.

which is in contradiction to the observed stoichiometry. Furthermore, the crystal structure clearly reveals three-dimensional interactions between the radical cations. This finding might explain the "smearing out" of sharp phase transitions.

\section{Physical Properties}

Fig. 2 shows the temperature dependence of the electrical resistivity of $(\mathrm{DIET})_{2}\left(\mathrm{BF}_{4}\right)_{3}$ as a typical example. The compound behaves as a metal at room temperature and below. At lower temperature, a very broad phase transition is indicated in the resistivity data. Fig. 3 summarizes results of the thermopower measurement on this material at different temperatures. The latter data clearly reveal a phase transition at around $50 \mathrm{~K}$. Similar results have been obtained from DIET salts with other anions like $\mathrm{HSO}_{4}{ }^{-}$or $\mathrm{NO}_{3}{ }^{-}$, as shown in Fig. 4 for the compound (DIET) $\left(\mathrm{NO}_{3}\right)_{y}$, the full structure of which could not be solved so far.

\section{CONCLUSIONS}

We have shown that is is possible to obtain organic metals from chiral molecules. The $\mathrm{X}$-ray results prove an oxidation number of +1.5 for the radical cations in at least two cases. To our best knowledge, these are the first examples of BEDT-TTF-related radical cation salts

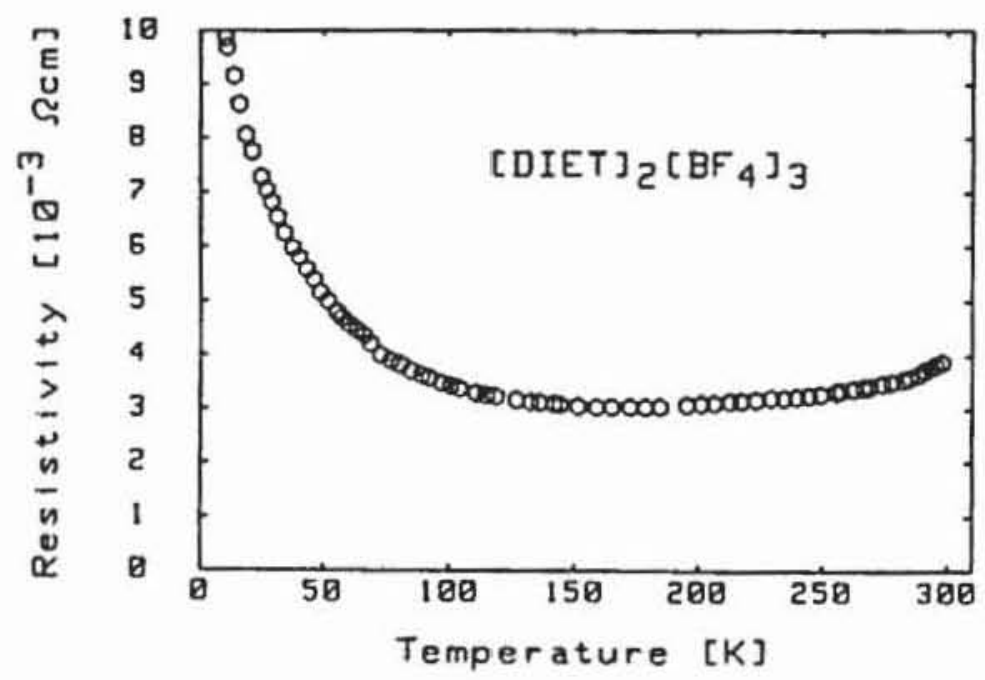

Fig. 2. Temperature dependence of the electrical resistivity of $(\mathrm{DIET})_{2}\left(\mathrm{BF}_{4}\right)_{3}$. 


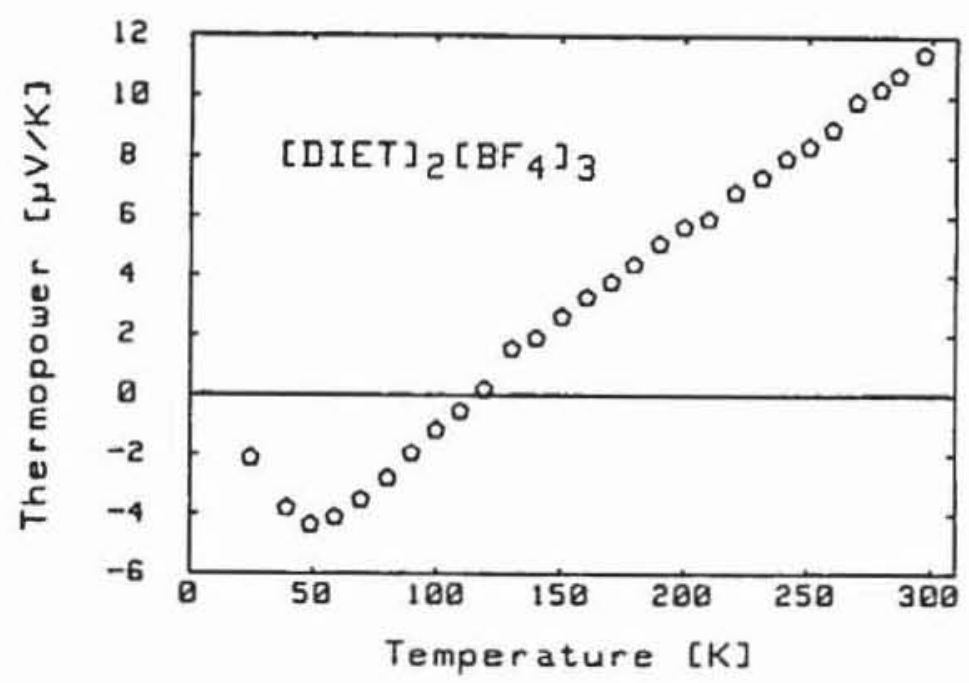

Fig. 3. Temperature dependence of the thermopower of $(\mathrm{DIET})_{2}\left(\mathrm{BF}_{4}\right)_{3}$.

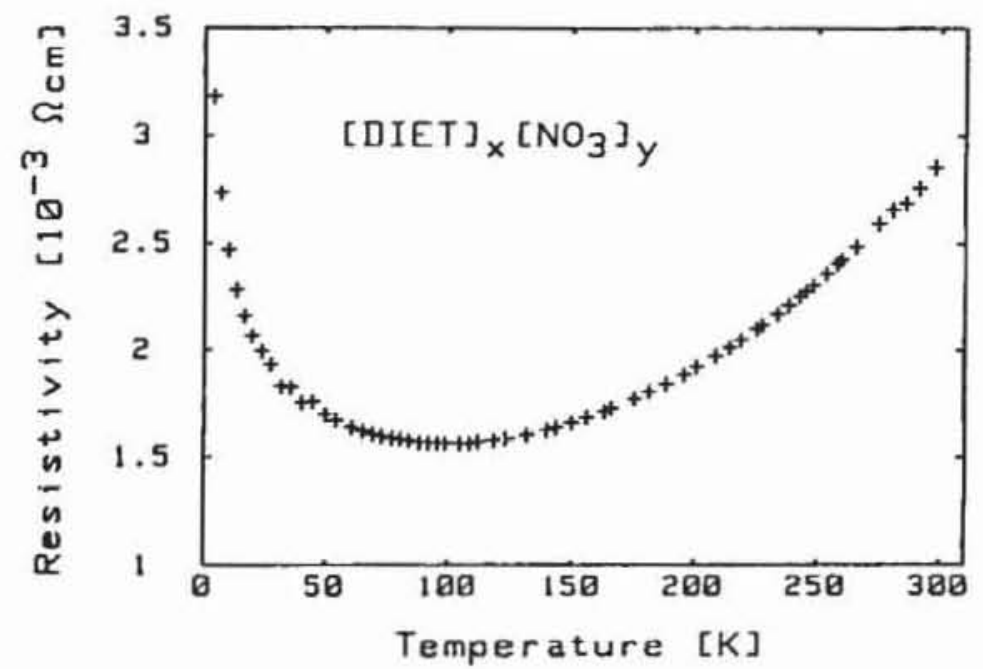

Fig. 4. Temperature dependence of the resistivity of $(\mathrm{DIET})_{\mathbf{x}}\left(\mathrm{NO}_{3}\right)_{\mathbf{y}}$.

with this oxidation number. As expected, we obtained statistically disordered crystals, containing the two different enantiomers in a "racemic" mixture. The broad smeared-out phase transitions are probably due to this disorder, or may be caused by the three-dimensional interactions which have been observed, so far, only once in a BEDT-TTF radical salt. We will crystallize the metallic compounds using "optically pure" isomers, in the hope to be able to isolate chiral metals.

\section{REFERENCES}

[1] See for example in: "Physical and Chemistry of Materials with Layered Structures," Vol. 1-5 (D. Reidel, Dordrecht, 1976).

[2] K. A. Müller and J. G. Bednorz, Z. Phys. B64: 189 (1986).

[3] J. M. Williams, H. H. Wang, T. J. Emge, U. Geiser, M. A. Beno, P. C. W. Leung, K. D. Carlson, R. J. Thorn, A. J. Schultz, and M.-H. Whangbo, Progr. Inorg. Chem. 30: 51 (1987).

[4] Proceedings of the ICSM 88, Santa Fe, Synth. Metals 27 (1989).

[5] G. Saito, T. Enoki, K. Toriumi, and H. Inokuchi, Solid State Commun. 42: 557 (1982). 
[6] G. C. Papavassilou, J. S. Zambounis, and S. Y. Yiannopoulos, Chem. Scripta 27: 261 (1987), and references cited therein.

[7] A. Karrer, J. D. Wallis, J. D. Dunitz, B. Hilti, C. W. Mayer, M. Bürkle, and J. Pfeiffer, Helv. Chim. Acta 70: 942 (1987).

[8] T. C. Umland, S. Allie, T. Kuhlmann, and P. Coppens, J, Phys, Chem., in print.

[9] U. Geiser, H. H. Wang, L. E. Gerdom, M. A. Firestone, L. M. Sowa, J. M. Williams, and M.-H. Whangbo, I. Am. Chem. Soc. 107: 8305 (1985). 